Dr. Roman Töppel, Historiker in München (romantoeppel@ hotmail.com) und Mitherausgeber der IfZ-Publikation „Hitler, Mein Kampf - eine kritische Edition“, veröffentlichte u.a.: „Die Sachsen und Napoleon. Ein Stimmungsbild 1806-1813“ (Köln/Weimar/Wien 22013); „Kursk - Mythen und Wirklichkeit einer Schlacht“, in: VfZ 57 (2009), S. 349-384; „Waffen-SS und Wehrmacht in der Schlacht bei Kursk. Ein Vergleich im operativen Einsatz", in: Jan Erik Schulte/Peter Lieb/Bernd Wegner (Hrsg.), Die Waffen-SS. Neue Forschungen (Paderborn 2014), S. 317-335.

Dr. Axel Schildt, Direktor der Forschungsstelle für Zeitgeschichte Hamburg (Beim Schlump 83, 20144 Hamburg) und Professor für Neuere Geschichte an der Universität Hamburg, veröffentlichte u. a.: „Die Sozialgeschichte der Bundesrepublik Deutschland bis 1989/90“ (München 2007); gemeinsam mit Detlef Siegfried „Deutsche Kulturgeschichte. Die Bundesrepublik - 1945 bis zur Gegenwart“ (München 2009); als Herausgeber mit Ute Daniel, „Massenmedien im Europa des 20. Jahrhunderts“ (Köln u.a. 2010); als Herausgeber mit Alexander Gallus und Detlef Siegfried, „Deutsche Zeitgeschichte - transnational“ (Göttingen 2015).

Dr. Mechthild Lindemann, wissenschaftliche Mitarbeiterin in der Abteilung „Akten zur Auswärtigen Politik der Bundesrepublik Deutschland“ (AAPD) des Instituts für Zeitgeschichte München-Berlin (c / o Auswärtiges Amt, Referat 117 - IfZ-AAPD 11013 Berlin); veröffentlichte u.a.: als Bearbeiterin zahlreiche AAPD-Bände, zuletzt AAPD 1962 und 1983 (München 2010 bzw. 2014); „Die deutsche Frage auf den Genfer Viermächtekonferenzen 1955“ (Bonn 1994); „Das Entschädigungsabkommen von 1961 zwischen der Schweiz und der Bundesrepublik Deutschland“, in: Die Schweiz und Deutschland 1945-1961, hrsg. von Antoine Fleury, Horst Möller und Hans-Peter Schwarz (München 2004), S. 147-163; „Ein Kasus als ,casus belli‘? Zum ,Genitiv-Streit‘ zwischen der Bundesrepublik Deutschland und der UdSSR in den 1970er und 1980er Jahren“, in: Irina Podtergera (Hrsg), Schnittpunkt Slavistik. Ost und West im wissenschaftlichen Dialog. Festgabe für Helmut Keipert zum 70. Geburtstag, Teil 3: Vom Wort zum Text (Göttingen 2012), S. 161-180.

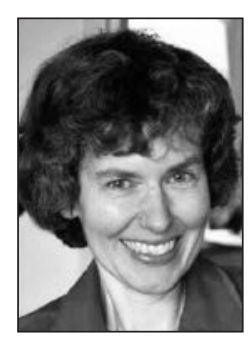




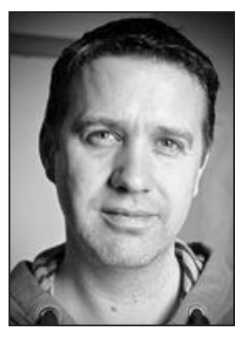

Dr. Thomas Riegler, Historiker und Redakteur (Vorgartenstraße 145-157/3/9, A-1020 Wien); veröffentliche u. a.: ,Terrorismus. Akteure, Strukturen, Entwicklungslinien“ (Innsbruck 2009); „Im Fadenkreuz: Österreich und der Nahostterrorismus 1973-1985“ (Vienna University Press 2010); „Die ,Wiener Residentur" der Stasi - Mythos und Wirklichkeit", in: Journal for Intelligence, Propaganda and Security Studies 7 (2013), Nr. 2, S. 89-113; „Agenten, Wissenschaftler und ,Todesstrahlen“: Zur Rolle österreichischer Akteure in Nassers Rüstungsprogramm (1958-1969)“, in: Ebenda 8 (2014), Nr. 2, S.44-72; „,Es muss ein gegenseitiges Geben und Nehmen sein“: Warschauer-PaktStaaten und Terrorismusbekämpfung am Beispiel der DDR“, in: Johannes Hürter (Hrsg.), Terrorismusbekämpfung in Westeuropa. Demokratie und Sicherheit in den 1970er und 1980er Jahren (Berlin u. a. 2014), S. 289-315.

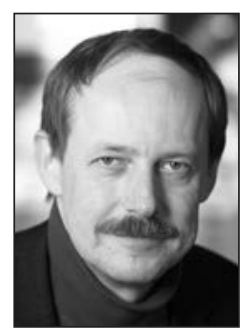

Dr. Frank Bajohr, Wissenschaftlicher Leiter des Zentrums für Holocaust-Studien am Institut für Zeitgeschichte, München (Leonrodstraße 46 b, 80636 München) und Privatdozent am Historischen Seminar der Universität Hamburg und Lehrbeauftragter am Historischen Seminar der Ludwig-MaximiliansUniversität München; veröffentlichte im letzten Jahr u.a.: als Herausgeber mit Andrea Löw, „Der Holocaust. Ergebnisse und neue Fragen der Forschung“ (Frankfurt a. M. 2015); als Herausgeber mit Jürgen Matthäus, „Alfred Rosenberg. Die Tagebücher 1934-1944“ (Frankfurt a. M. 2015); als Herausgeber mit Sybille Steinbacher, „,Zeugnis ablegen bis zum letzten“. Tagebücher und persönliche Zeugnisse aus der Zeit des Nationalsozialismus und des Holocaust" (Göttingen 2015).

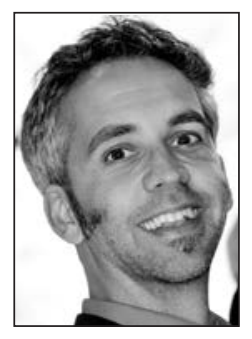

Dr. Michael Mayer, zuständig für den Arbeitsbereich Zeitgeschichte an der Akademie für Politische Bildung Tutzing (Buchensee 1, 82327 Tutzing); veröffentlichte u.a.: „Deformation der Gesellschaft. Neue Forschungen zum Nationalsozialismus“ (Berlin 2008); „Staaten als Täter. Ministerialbürokratie und ,Judenpolitik" in NS-Deutschland und Vichy-Frankreich. Ein Vergleich“ (München 2010); „,Die französische Regierung packt die Judenfrage ohne Umschweife an'. Vichy-Frankreich, deutsche Besatzungsmacht und der Beginn der ,Judenpolitik“ im Sommer /Herbst 1940“, in: VfZ 58 (2010), S. 329-362; „Akteure, Verbrechen und Kontinuitäten. Das Auswärtige Amt im Dritten Reich. Eine Binnendifferenzierung“, in: VfZ 59 (2011), S. 509-532; als Bearbeiter „Die Verfolgung und Ermordung der 


\section{Autoren 189}

europäischen Juden durch das nationalsozialistische Deutschland 1933-1945, Bd. 5: West- und Nordeuropa 1940 bis Juni 1942“ (München 2012); als Herausgeber mit Johannes Hürter, „Das Auswärtige Amt in der NS-Diktatur“ (München 2014); als Bearbeiter die AAPD-Bände 1978 (2 Bände) und 1962 (3 Bände) (München 2009/2010). 


\section{DE}

DE GRUYTER

OLDENBOURG

QUELLEN UND

DARSTELLUNGEN ZUR

ZEITGESCHICHTE

\section{BAND 109}

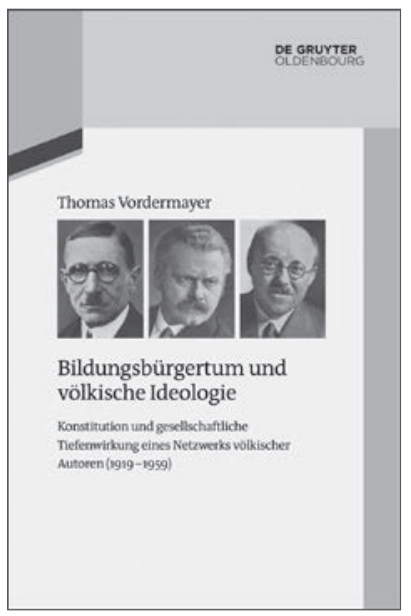

Thomas Vordermayer

Bildungsbürgertum und völkische Ideologie

Konstitution und gesellschaftliche Tiefenwirkung eines

Netzwerks völkischer Autoren (1919-1959)

2016, X, 469 S.

Ln. $€ 59,95$

ISBN 978-3-11-041475-2

eBook $€ 59,95$

PDF ISBN 978-3-11-041553-7

ePUB ISBN 978-3-11-041569-8

Print + eBook $€ 89,95$

ISBN 978-3-11-041554-4

Die gemäßigt agierenden völkischen Ideologen Hans Grimm, Erwin Guido Kolbenheyer und Wilhelm Stapel beeinflussten die bildungsbürgerlichen Eliten ihrer Zeit in einer Weise, die weniger distinguiert auftretenden völkischen Agitatoren verschlossen blieb. Thomas Vordermayer zeichnet die Karrieren der drei Erfolgsautoren zwischen 1919 und 1959 nach und zeigt, wie sie unter den politisch-ideologischen »Multiplikatoren« der deutschen Gesellschaft Deutungsmacht erlangten. Dem Leser eröffnen sich dadurch ganz neue Perspektiven auf die ideologische Verführbarkeit des Weimarer Bildungsbürgertums sowie auf das Denken und Handeln völkischer Schriftsteller und Publizisten. Wie sie sich untereinander abstimmten und bestätigten, wie sie sich im »Dritten Reich« positionierten und wie sie ihren jähen Bedeutungsverlust nach 1945 mental verarbeiteten, ist noch nie so nuanciert und tiefgründig beschrieben worden, wie in dieser preisgekrönten Studie.

Thomas Vordermayer ist wissenschaftlicher Mitarbeiter am Lehrstuhl für Neuere und Neueste Geschichte der Universität München. 